\title{
THE EFFECTS OF A FOUR WEEK JUMP-TRAINING PROGRAM ON FRONTAL PLANE PROJECTION ANGLE IN FEMALE GYMNASTS
}

\author{
Colclough, A. ${ }^{\text {a }}$ Munro, AG. ${ }^{a}$ Herrington, LC. ${ }^{a}$ McMahon, JJ. ${ }^{a} \&$ \\ Comfort, $\mathrm{P}^{\mathrm{a}^{*}}$
}

${ }^{a}$ Directorate of Sport, Exercise and Physiotherapy, University of Salford, Frederick Road, Salford, Greater Manchester. M6 6PU. United Kingdom.

*Corresponding author: p.comfort@salford.ac.uk 


\section{ABSTRACT}

Objectives: To investigate the effects of a four week jump training program on frontal plane projection angle (FPPA) in young female gymnasts.

Design: Intervention study, consisting of a four week jump training program performed for 15 minutes as part of a warm-up, three days per week for four weeks.

Setting: Gymnastics training center.

Participants: Fourteen youth female gymnasts (age: $13.5 \pm 2.14$ years, height: $1.54 \pm 0.11 \mathrm{~m}$, body mass: $46.23 \pm 7.68 \mathrm{~kg}$ ).

Main Outcome Measures: Change in FPPA during a $30 \mathrm{~cm}$ drop landing and tuck back somersault.

Results: Large and significant decreases $(\mathrm{p}<0.001)$ in FPPA of $6.8^{\circ}(39 \%)$ and $8.4^{\circ}(37 \%)$ during the drop landing and tuck back somersault, respectively.

Conclusion: The jump training program was successful in improving FPPA in female gymnasts and is advised to be implemented into the warm-ups and training programs of competitive female gymnasts to improve FPPA and therefore reduce the risk factors associated with knee injuries.

Keywords: Landing mechanics; Lower limb alignment; Valgus; Injury prevention

\section{Highlights:}

- FPPA is highly reliable during a $30 \mathrm{~cm}$ drop landing

- FPPA is highly reliable during landing from a tuck back somersault

- Regular jump training incorporated into a warm-up improves FPPA in young female gymnasts 


\section{INTRODUCTION}

The exposure of female athletes to a high frequency of high force landings during sports participation increases lower limb injury risk (Griffin et al., 2000; Griffin et al., 2006; Hewett, Ford, Hoogenboom, \& Myer, 2010). The majority of these injuries occur within the knee joint, often resulting in season-ending injuries such as anterior cruciate ligament (ACL) tears and patellofemoral pain syndrome (Griffin et al., 2000; Hewett et al., 2005).

Video analysis of injury situations reveals that excessive loading at the knee joint results in high valgus (abduction) angles at the knee, which is reported to be a primary predictor of knee injuries in female athletes (Hewett et al., 2010; Hewett et al., 2005; Marinsek, 2010; Otsuki, Kuramochi, \& Fukubayashi, 2014). Research has widely used the "gold standard" three dimensional (3D) motion analysis of lower limb kinematics for the measurement of knee valgus due to its accurate and reliable nature (Ford, Myer, \& Hewett, 2003; Hewett et al., 2005; McLean et al., 2005). This technique, however, is costly and time consuming and may be unsuitable in large scale screening programs as well as being inaccessible to many sports coaches (Ekegren, Miller, Celebrini, Eng, \& Macintyre, 2009; McLean et al., 2005). Researchers have therefore developed a simple, less expensive and easy-to-use two dimensional (2D) method for the analysis of lower limb alignment, measuring frontal plane projection angle (FPPA) (McLean et al., 2005; Munro, Herrington, \& Carolan, 2012; Wilson \& Davis, 2008). This 2D method has been reported to provide reliable measures of FPPA for movements that occur in the frontal plane (Munro et al., 2012) and can be used to successfully screen people at risk of injuries that arise from dynamic knee valgus angles (McLean et al., 2005). More recently this method was also reported to be reliable in assessing FPPA during drop landings and landing during a tuck back somersault in female gymnasts (Comfort, Colclough, \& Herrington, 2016). 
The knee and ankle account for $34 \%$ of injuries in female gymnasts (Westermann, Giblin, Vaske, Grosso, \& Wolf, 2015), with the ACL being the most commonly reported knee injury (Majewski, Susanne, \& Klaus, 2006). The nature of gymnastics involves a high frequency of jump landings in which athletes must control the rotation of the body in flight and absorb high ground reaction forces upon landing. Concurrently, gymnasts must ensure contact with the ground is made safely and effectively (Marinsek, 2010), but they are encouraged not to land with a high degree of flexion at the knee, as this incurs increased point deductions in competition. Reduced knee flexion during landing is ideal for high scores based on the scoring criteria within gymnastics, but this may result in increased forces at landing and add to ACL strain, which may be a contributing factor to knee injuries (Marinsek, 2010; Utturkar et al., 2013). Additionally, high FPPA angles have been reported in young female gymnasts during jump landings and landing from a tuck back somersault (Comfort et al., 2016) which may partially be a compensatory strategy for the limited knee flexion in order to attenuate the landing forces.

Jump training programs have been reported to improve dynamic knee valgus, FPPA and decrease ground reaction forces during landings, by prompting athletes to focus on knee alignment and technique improvement during exercises (Aerts, Cumps, Verhagen, \& Meeusen, 2010; Bien, 2011; Herrington, 2010; Herrington, Munro, \& Comfort, 2015; Hewett, Lindenfeld, Riccobene, \& Noyes, 1999). The approaches to the reported jump training programs in the literature have been varied. The majority of the reported training programs have been 4-8 weeks in duration and have involved 3 sessions per week of around 10-20 minutes (Herrington, 2010; Herrington et al., 2015; Kato, Urabe, \& Kawamura, 2008). Some programs may last up to 6 months; involve 6 days per week, for a duration of 90 minutes per day (Chappell \& Limpisvasti, 2008; Myer, Ford, Palumbo, \& Hewett, 2005; Otsuki et al., 2014), although these are less likely to be implemented successfully in most 
sports due to the constraints of training time. More recently, warm-up style jump training programs of 10-15 minutes have become popular among sports practitioners for their practicality and minimal time commitments (Herrington, 2010; Herrington et al., 2015; Herrington, Myer, \& Horsley, 2013; Kato et al., 2008).

In terms of training content, many researchers have incorporated jump training with additional exercise foci such as balance, strength, stretching and flexibility (Hewett et al., 1999; Hewett, Stroupe, Nance, \& Noyes, 1996; Myer et al., 2005; Paterno, Myer, Ford, \& Hewett, 2004). The study by Herrington (2010) is the only study to investigate the impact of a 4 week training program using jumping alone, to improve FPPA. The results of his study had similar positive effects upon landing strategies when compared to longer duration, mixed-content training programs with FPPA for the drop jump landing task being reduced by an average of $57 \%\left(12.3^{\circ}\right)$ and $73 \%\left(9.8^{\circ}\right)$ for the right and left leg, respectively.

While high FPPA have been reported in young female gymnasts (Comfort et al., 2016) currently no researchers appear to have investigated the effects of injury prevention training programs on FPPA in female gymnasts. This is surprising, considering the fact that gymnasts perform multiple landings, consisting of high loads, great heights, and frequent rotation of the body in excess of 200 times within the typical training week (Marinsek, 2010). Therefore, the aim of this study was to determine the effects of a brief jump training program integrated into a warm-up (Herrington, 2010) on FPPA during a bilateral drop landing task and during landing from a tuck back somersault, in female gymnasts. It was hypothesized that 4 weeks of jump training would decrease FPPA observed during both landing tasks, in line with previous research in female basketball players (Herrington, 2010). 


\section{METHODS}

\section{Experimental approach}

A 4 week progressive jump training intervention, incorporated into the participant's warmup, was conducted to assess the effects of jump landing on FPPA in female gymnasts. Using methods previously reported by Munro et al. (2012) and Wilson and Davis (2008), 2D measurement of FPPA was assessed across two different landing tasks (drop landing and tuck back somersault). Baseline FPPA data was collected twice pre-intervention, one week before the initial intervention session, with a follow up FPPA data collection post-intervention, within one week of concluding the final session, resulting in three data collection session (two pre intervention, one post intervention). This established reliability of the measures and determined the effects of the intervention.

\section{$\underline{\text { Participants }}$}

Adolescent, female competitive (competed $\geq 6$ times in the previous 12 months) gymnasts (n $=14$, age $13.5 \pm 2.14$ years, range $12-17$ years; height $1.54 \pm 0.11 \mathrm{~m}$, range $1.38-1.67 \mathrm{~m}$; body mass $46.23 \pm 7.68 \mathrm{~kg}$, range $35-56.51 \mathrm{~kg}$ ), who train $\sim 15$ hours per week volunteered to participate in the study. All participants had no history of ACL injury or other knee pathology and were free from any lower extremity injury for 6 months prior to data collection. All participants completed a minimum of $90 \%$ of the jump training sessions (11 of 12) and training took place at the same time of day in the same gymnastics training center for the duration of the program. Consent was obtained from all participants with further written consent from parents or guardians as appropriate. 


\section{$\underline{\text { Testing Protocols }}$}

Participants FPPA were assessed before and after the intervention period and participants were asked to refrain from strenuous exercise for 24 hours prior to testing. To simulate the landings encountered during gymnastics, the participants were asked to perform a $30 \mathrm{~cm}$ bilateral drop landing and a tuck back somersault. To familiarize participants with each task, they performed 5 practice trials of the 2 tasks, as part of their warm-up. Participants then performed 3 test trials for each task with a rest period of $30 \mathrm{~s}$ between each repetition and 60 $\mathrm{s}$ between each task. The landing surface for the 2 tasks was identical $(20 \mathrm{~cm}$ shockabsorbent landing mat) and trials were only accepted if the gymnast landed on both feet with no additional foot movement permitted. The participants performed the tasks in bare feet, with no taping or bracing of the joints. All equipment used in the test complied with the safety standards of the testing venue which follows the safety standards as set per the International Gymnastics Federation (FIG).

Prior to performing the tasks pen marks were made on the center of the ankle (mid-point between the medial and lateral malleoli), patella (mid-point between the medial and lateral epicondyle) and mid-way up the center of the thigh (between the patella marker and the anterior superior iliac spine) (Munro et al., 2012). These markers then permitted calculation of the resultant FPPA described later.

Bilateral drop landing $(30 \mathrm{~cm})$ : Participants stood on a $50 \mathrm{~cm}$ box with their feet shoulder width apart, dropped off the box onto a $20 \mathrm{~cm}$ shock absorbent landing mat (resultant drop height $30 \mathrm{~cm}$ ), landing on a tape marker placed $30 \mathrm{~cm}$ in front of the box. The participants were asked to not jump or step down off the box and were asked to perform the task naturally, with no set instructions regarding arm movement. If these instructions were not followed or the participant did not stick the landing they were ask to repeat the trial, after $30 \mathrm{~s}$ of rest. 
Tuck back somersault: Participants stood on a tape marker placed on the shock absorbent mat and in their own time performed a standing tuck back somersault, landing with both feet on a separate tape marker, $30 \mathrm{~cm}$ behind the initial marker. If the marker was missed or the participant did not stick the landing they were ask to repeat the trial, after $30 \mathrm{~s}$ of rest.

Two dimensional FPPA of knee valgus alignment was measured during the two tasks using the methods performed by Munro et al. (2012) and Wilson and Davis (2008) pre and post intervention. A high definition digital video camera (Casio Exilim EX-F1) was mounted at the height of the subject's knee (individually measured from the floor to the midpoint of the patella with the participant standing), $2 \mathrm{~m}$ anterior to the landing target in the frontal plane. A one square meter calibration frame was videoed before each subject performed each task to allow for subsequent calibration of the video footage in order to yield accurate resultant angles. A still image, representing the lowest point of the landing based on the displacement of the marker on the patella, was taken from the video and imported into a digitizing software program (ImageJ for Windows, 1.46R, USA). A single experimenter measured and calculated the angles. Calculation of the FPPA involved drawing a line from the marker at the anterior superior iliac spine and the marker at the middle of the tibiofemoral joint and another line from this point to the marker at the midpoint of the ankle malleoli, with the angle calculated via the software, in line with previous research (Comfort et al., 2016; Munro et al., 2012).

\section{Training Intervention}

The subjects undertook a four week progressive jump training program (Table 1) that incorporated bilateral, unilateral and directional control landings (Herrington, 2010). Emphasis was placed on the quality of movements, with verbal feedback provided by 
coaching staff, highlighting proper knee alignment and technique perfection. The jump training program was incorporated into the warm-up, lasted 15 minutes and was performed three times per week, prior to their sports specific warm up. All subjects completed $100 \%$ of the jump training sessions.

Table 1. Jump Training Program

\begin{tabular}{|c|c|c|}
\hline WEEK 1 & & \\
\hline SESSION 1 & SESSION 2 & SESSION 3 \\
\hline Wall jumps - $20 \mathrm{~s}$ & Wall jumps - $20 \mathrm{~s}$ & Wall jumps - $20 \mathrm{~s}$ \\
\hline Tuck jumps - $20 \mathrm{~s}$ & Tuck jumps - $20 \mathrm{~s}$ & Tuck jumps - $20 \mathrm{~s}$ \\
\hline Broad jumps* $5 \times 3$ & Broad jumps* $5 \times 3$ & Broad jumps $* 5 \times 5$ \\
\hline Squat jumps 5 × 3 & Squat jumps $5 \times 3$ & Squat jumps $5 \times 5$ \\
\hline Forward jumps (barrier) $5 \times 3$ & Forward jumps (barrier) $-5 \times 3$ & Forward jumps (barrier) $-7 \times 3$ \\
\hline $180^{\circ}$ Jumps - 10 & $180^{\circ}$ Jumps - 10 & $180^{\circ}$ Jumps -10 \\
\hline Lunge walk $2 \times 15 \mathrm{~m}$ & Lunge walk $2 \times 15 \mathrm{~m}$ & Lunge walk $1 \times 15 \mathrm{~m}$ \\
\hline WEEK 2 & & \\
\hline SESSION 1 & SESSION 2 & SESSION 3 \\
\hline Wall jumps - $20 \mathrm{~s}$ & Wall jumps - $20 \mathrm{~s}$ & Wall jumps - $20 \mathrm{~s}$ \\
\hline Tuck jumps - $20 \mathrm{~s}$ & Tuck jumps $-20 \mathrm{~s}$ & Tuck jumps $-20 \mathrm{~s}$ \\
\hline Broad jumps* -5 x 5 & Broad jumps distance* $-5 \times 3$ & Broad jumps distance* $-5 \times 5$ \\
\hline Squat jumps $-5 \times 5$ & Jump, jump, vertical - 5 × 3 & Jump, jump, vertical - 5 x 5 \\
\hline Forward jumps (barrier) $7 \times 3$ & Side jumps (barrier) $5 \times 3$ & Side jumps (barrier) 7 × 3 \\
\hline $180^{\circ}$ Jumps -10 & $180^{\circ}$ Jumps -10 & $180^{\circ}$ Jumps - 10 \\
\hline Lunge walk 1 x $15 \mathrm{~m}$ & Split lunge $3 \times 4$ & Split lunge $3 \times 6$ \\
\hline WEEK 3 & & \\
\hline SESSION 1 & SESSION 2 & SESSION 3 \\
\hline Wall jumps $-20 \mathrm{~s}$ & Wall jumps - $20 \mathrm{~s}$ & Wall jumps - $20 \mathrm{~s}$ \\
\hline Tuck jumps - $20 \mathrm{~s}$ & Tuck jumps - $20 \mathrm{~s}$ & Tuck jumps - $20 \mathrm{~s}$ \\
\hline Hop 5 x 3 & Hop 5 x 5 & Hop 5 x 5 \\
\hline Jump, jump, vertical $\left(180^{\circ}\right) 5 \times 3$ & Jump, jump, vertical $\left(180^{\circ}\right) 5 \times 5$ & Jump, jump, single land $5 \times 6$ \\
\hline Forward hops (barrier) $5 \times 3$ & Forward hops (barrier) 7 x 3 & Side hops (barrier) 5 x 3 \\
\hline $270^{\circ}$ Jumps -10 & $270^{\circ}$ Jumps -10 & $90^{\circ}$ Hops -10 \\
\hline Split lunge $3 \times 6$ & Split lunge $3 \times 8$ & Split lunge $3 \times 8$ \\
\hline WEEK 4 & & \\
\hline SESSION 1 & SESSION 2 & SESSION 3 \\
\hline Wall jumps $-20 \mathrm{~s}$ & Wall jumps - $20 \mathrm{~s}$ & Wall jumps $-20 \mathrm{~s}$ \\
\hline Tuck jumps - $20 \mathrm{~s}$ & Tuck jumps - $20 \mathrm{~s}$ & Tuck jumps - $20 \mathrm{~s}$ \\
\hline Hop 6 × 5 & Crossover Hop 6 × 3 & Crossover Hop 6 x 3 \\
\hline Jump, jump, single land* 6 x 6 & Jump, jump, single land, 6 x 4 & Jump, jump, single land $6 \times 4$ \\
\hline Side hops (barrier) 7 × 3 & Side hops (barrier) 7 × 3 & Side hops (barrier) 7 × 3 \\
\hline $90^{\circ}$ Hops -10 & $180^{\circ}$ Hops - 6 & $180^{\circ}$ Hops -6 \\
\hline Split lunge $90^{\circ} 3 \times 6$ & Split lunge $90^{\circ} 3 \times 6$ & Split lunge $90^{\circ} 3 \times 6$ \\
\hline
\end{tabular}




\section{$\underline{\text { Statistical Analyses }}$}

Means and standard deviations were calculated for all FPPA values and distribution of data was assessed via Shapiro-Wilks' tests of normality. Intraclass correlation coefficients $(2,3$ (ICC)) were calculated to determine between-session reliability. The ICC's were interpreted based on the recommendations of Hopkins (2002), where $<0.10,0.10-0.29,0.30-0.49,0.50-$ $0.69,0.70-0.89$ and $\geq 0.9$ are considered insubstantial, low, moderate, high, very high and nearly perfect.

Paired samples t-tests or Wilcoxon signed ranks tests were used, based on normal distribution of the data, to identify differences in FPPA between the two baseline testing days, to determine if there was any learning effect, as well as pre-and post-intervention to determine the effect of the intervention, and to determine the magnitude of change between limbs, using peak values for both tasks. Effect sizes were also calculated to determine the magnitude of any observed differences using the Cohen's $d$ method and interpreted based on the recommendations of Rhea (2004), where $<0.50,0.50-1.25,1.25-1.9$, and $>2.0$, are considered trivial, small, moderate and large for recreationally trained athletes. Data analyses were conducted using SPSS for Windows (Version 22; IBM, USA). An a priori alpha level was set at $p<0.05$.

\section{RESULTS}

Pre-intervention FPPA showed a high to very high reliability and low measurement error during the drop landing and tuck back somersault (Table 2). Additionally, Shapiro-Wilk's test of normality revealed FPPA values were not normally distributed for the three testing days ( $p$ $>0.05$ ), with the results of Wilcoxon signed ranks test demonstrating no significant, trivial 
differences ( $\mathrm{p}>0.05$, Cohen's $d \leq 0.16)$ in FPPA between baseline testing days (Day 1 and 2), demonstrating no systematic learning effect.

Table 2: Descriptive statistics (mean \pm standard deviation) and between session reliability (ICC) and measurement error (SEM and SDD)

\begin{tabular}{l|ccccc}
\multicolumn{1}{l}{ Task } & Day 1 & Day 2 & ICC & SEM & SDD \\
\hline Drop Landing & $16.2 \pm 5.9^{\circ}$ & $17.2 \pm 6.2^{\circ}$ & 0.68 & $1.63^{\circ}$ & $4.5^{\circ}(27 \%)$ \\
Tuck back somersault & $22.1 \pm 7.5^{\circ}$ & $22.8 \pm 7.2^{\circ}$ & 0.87 & $0.81^{\circ}$ & $2.3^{\circ}(10 \%)$
\end{tabular}

The results of Wilcoxon signed ranks tests revealed a large and significant decrease $(\mathrm{p}<0.001$; Cohen's $d=1.17 ; 39 \%)$ in FPPA during the drop landing pre $\left(17.3 \pm 6.2^{\circ}\right)$ to post $(10.5 \pm$ $5.4^{\circ}$ ) intervention. A similarly large significant decrease ( $\mathrm{p}<0.001$; Cohen's $d=1.35 ; 37 \%$ ) was observed for FPPA during the tuck back somersault pre $\left(22.8 \pm 7.2^{\circ}\right)$ to post $(14.4 \pm$ $5.1^{\circ}$ ) intervention (Figure 1), with both improvements in FPPA greater than the SDD.

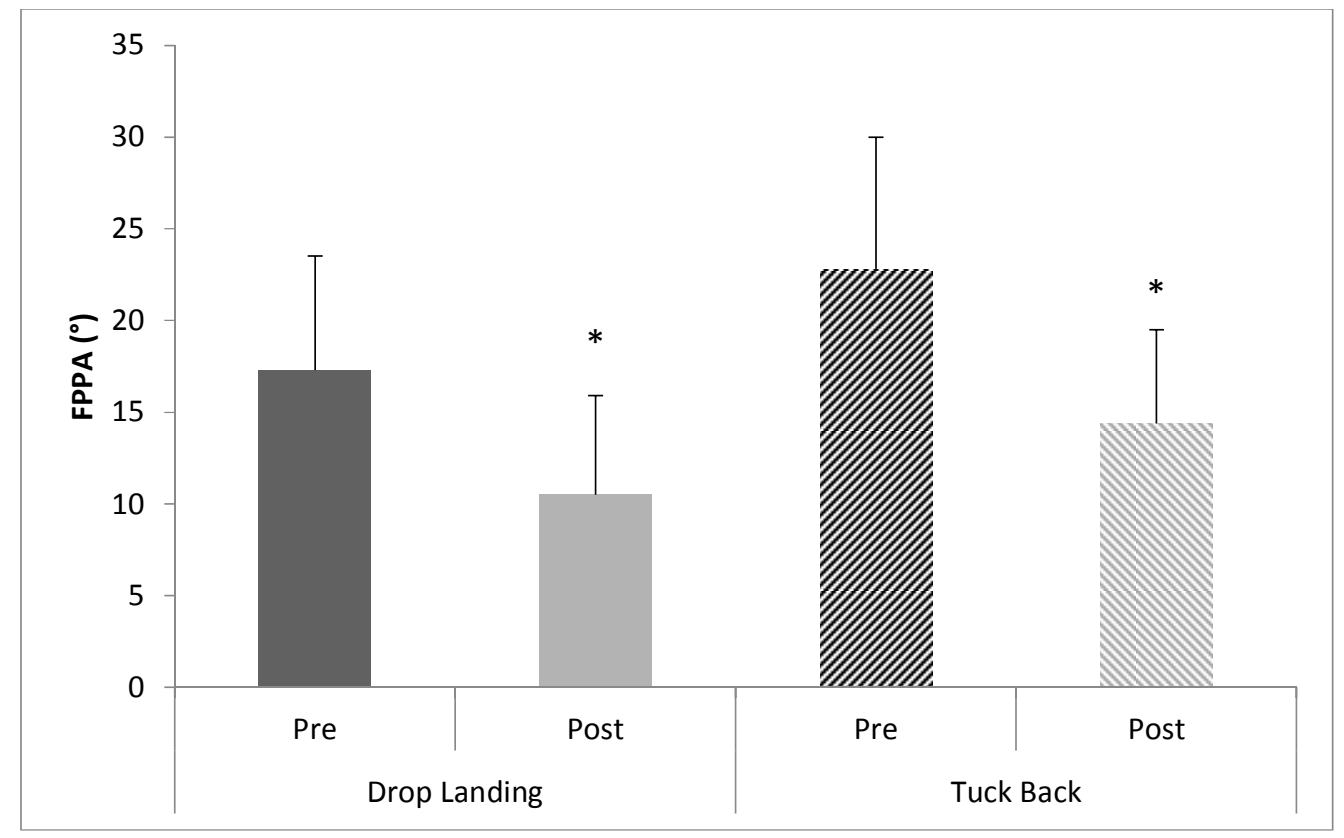

Figure 1: Comparison of FPPA pre and post intervention (* significantly less $(\mathrm{p}<0.001)$ than pre intervention) 


\section{DISCUSSION}

The female gymnasts within this study demonstrated high baseline FPPAs across both landing tasks (drop landing $17.3 \pm 6.2^{\circ}$; tuck back somersault $22.8 \pm 7.2^{\circ}$ ), when compared to previously reported drop landing values for a physically active population (8.2-9.9 $)(10)$, with the more functional and demanding task, the tuck back somersault, revealing the greatest FPPA values. Furthermore, as hypothesized the results demonstrate that a 4 week jump training program induces significant improvements in FPPA in female gymnasts. The intervention resulted in a large meaningful 39\% decrease in FPPA for the drop landing task and a decrease of $37 \%$ for the tuck back somersault, which was greater than the between session SDDs.

Landing technique in women's gymnastics, in terms of knee valgus and FPPA, has received limited research, even though the ACL has been reported to be the most common site of knee injury (Majewski et al., 2006). The large FPPA baseline values reported in this study (drop landing $17.3 \pm 6.2^{\circ}$; tuck back somersault $22.8 \pm 7.2^{\circ}$ ), are similar to those that we previously reported in young female gymnasts (drop landing 12.2-19.6 ${ }^{\circ}$; tuck back somersault $\left.16.1-20.3^{\circ}\right)($ Comfort et al., 2016). More importantly, these baseline values are greater than the normative FPPA values previously reported by Herrington and Munro (2010) who suggest that individuals who demonstrate FPPAs greater than $7-13^{\circ}$ for a drop landing task may be at risk of injury to the patellofemoral joint or ACL (McLean et al., 2005; Wilson \& Davis, 2008). The high FPPA values reported in the current study may be due to the exposure of gymnasts to a high frequency of high force landings, on a compliant surface, on a regular basis (Gittoes \& Irwin, 2012). Such landings, combined with a stiff landing strategy on a complaint surface may result in forces being attenuated through the adverse frontal plane movement. Repeated stress on the lower extremity may increase the FPPA at the knee joint and increase the risk of ACL injuries in female gymnasts (Marinsek, 2010). 
Previous research suggests that jump training is an integral feature of training programs for the reduction of ACL injury risk (Herrington, 2010; Herrington et al., 2015; Hewett et al., 1999; Hewett et al., 1996; Myer, Ford, Brent, \& Hewett, 2012; Myer et al., 2005). Training interventions that incorporate a jump element and focus on knee alignment during landing may induce neuromuscular adaptations in female athletes and prepare them for functional jumping tasks during sporting activities (Hewett et al., 2010; Myer et al., 2012). Herrington, Munro and Comfort (2015) suggest that jump training relies on skill acquisition by educating the neuromuscular system to react in an appropriate way to carry out the landing task.

The improvements in FPPA (drop landing $17.3 \pm 6.2^{\circ}$; tuck back somersault $22.8 \pm 7.2^{\circ}$ ) observed during the current study were lower than those observed during a drop landing task in the study of Herrington (2010) (57\% and 73\% for the right and left legs, respectively). As the same training intervention was used it is likely that the age and development of the athletes in this study and the use of a compliant landing surface may partly explain some of the differences in the magnitude of the changes in FPPA.

It is important to determine the demands and potential risks associated with different athletic tasks, as Cowley et al. (2006) suggest that individual tasks can influence the amount of load that occurs at the knee during a landing task, with, for example, cutting tasks reported to at least double the valgus load compared to straight running. In gymnastics, skills performed from the asymmetric bars and the floor apparatus will vary in rotation of the body, flight, speed and ground reaction force. Therefore some tasks may double the valgus load of the knee compared to others (Cowley et al., 2006). Forces measured at landing can range from 3.9 to 14.4 times body weight (Marinsek, 2010), hence, the tuck back somersault landing task was used in this study as a functional task to present the element of backwards rotation and increase the force absorbed by the lower extremities upon impact with the floor, as well as providing a more ecologically valid result to assess the effectiveness of the training program. 
Hence, future research ought to investigate different gymnastics elements with high forces at landing to examine their effects on FPPA. In the current study FPPA showed a large and meaningful decrease (Cohen's $d=1.35 ; 37 \%$ ) during the tuck back somersault. In comparison, Kato et al. (2008) and Herrington (2010) used a jump shot landing as a functional task in their study to obtain a more ecological result in female basketball players. The results are in line with those of the current study, with the study of Kato et al. (2008) revealing an average $41 \%$ decrease in FPPA while Herrington (2010) revealed a 36\% decrease in FPPA during the functional jump shot landing task. It is clear that jump training that focuses on lower limb alignment during landing tasks has the potential to significantly improve FPPA during a standard drop landing task as well as a more complex functional task.

Two potential limitations of this study are the landing being performed on a compliant surface and the use of FPPA rather than knee valgus assessed via 3D motion analysis. However, a shock absorbent landing mat was used to imitate landing conditions in training and competition because any change in landing surface, such as a force plate would be likely to alter landing mechanics substantially and therefore reduce ecological validity. While FPPA has not been directly linked to the risk factors associated with ACL injuries, it has been significantly correlated with hip adduction $(r=0.32, p=0.044)$ and knee external rotation $(r$ $=0.48, \mathrm{p}=0.002$ ), both of which are components of knee valgus (McLean et al., 2005; Munro et al., 2012; Wilson \& Davis, 2008). Researchers have reported that FPPA may possess the potential to successfully screen people at risk of injuries that arise from dynamic knee valgus angles (McLean et al., 2005; Munro et al., 2012; Wilson \& Davis, 2008). 


\section{Conclusion}

A short duration jump landing training program can have positive effects in reducing FPPA in female gymnasts. A progressive jump training program implemented into the warm-up, 3 times per week allows gymnasts to learn correct landing techniques from an early age and physically prepare themselves to overcome various loads at landing in a safe and effective way. Due to the high baseline FPPA values reported in this study, individuals involved in training these athletes ought to consider this injury prevention technique in order to reduce the risk factors associated with ACL injuries, which should not impact negatively on the aesthetic aspects of the landing or therefore the scores awarded. 


\section{REFERENCE LIST}

Aerts, I., Cumps, E., Verhagen, E., \& Meeusen, R. (2010). Efficacy of a 3 month training program on the jump-landing technique in jump-landing sports. Design of a cluster randomized controlled trial. BMC Musculoskelet Disord, 11, 281.

Bien, D. P. (2011). Rationale and implementation of anterior cruciate ligament injury prevention warm-up programs in female athletes. J Strength Cond Res, 25(1), 271-285.

Chappell, J. D., \& Limpisvasti, O. (2008). Effect of a Neuromuscular Training Program on the Kinetics and Kinematics of Jumping Tasks. The American Journal of Sports Medicine, 36(6), 10811086.

Comfort, P., Colclough, A., \& Herrington, L. (2016). A Comparison of Frontal Plane Projection Angle Across Landing Tasks in Female Gymnasts. International Journal of Athletic Therapy \& Training, 21(5), 42-47.

Cowley, H. R., Ford, K. R., Myer, G. D., Kernozek, T. W., \& Hewett, T. E. (2006). Differences in neuromuscular strategies between landing and cutting tasks in female basketball and soccer athletes. J AthI Train, 41(1), 67-73.

Ekegren, C. L., Miller, W. C., Celebrini, R. G., Eng, J. J., \& Macintyre, D. L. (2009). Reliability and validity of observational risk screening in evaluating dynamic knee valgus. J Orthop Sports Phys Ther, 39(9), 665-674.

Ford, K. R., Myer, G. D., \& Hewett, T. E. (2003). Valgus knee motion during landing in high school female and male basketball players. Med Sci Sports Exerc, 35(10), 1745-1750.

Gittoes, M., Jr., \& Irwin, G. (2012). Biomechanical approaches to understanding the potentially injurious demands of gymnastic-style impact landings. Sports Med Arthrosc Rehabil Ther Technol, 4(1), 4.

Griffin, L. Y., Agel, J., Albohm, M. J., Arendt, E. A., Dick, R. W., Garrett, W. E., et al. (2000). Noncontact anterior cruciate ligament injuries: risk factors and prevention strategies. J Am Acad Orthop Surg, 8(3), 141-150.

Griffin, L. Y., Albohm, M. J., Arendt, E. A., Bahr, R., Beynnon, B. D., Demaio, M., et al. (2006). Understanding and preventing noncontact anterior cruciate ligament injuries: a review of the Hunt Valley II meeting, January 2005. Am J Sports Med, 34(9), 1512-1532.

Herrington, L. (2010). The Effects of 4 Weeks of Jump Training on Landing Knee Valgus and Crossover Hop Performance in Female Basketball Players. The Journal of Strength \& Conditioning Research, 24(12), 3427-3432.

Herrington, L., \& Munro, A. (2010). Drop jump landing knee valgus angle; normative data in a physically active population. Physical Therapy in Sport, 11(2), 56-59.

Herrington, L., Munro, A., \& Comfort, P. (2015). A preliminary study into the effect of jumpinglanding training and strength training on frontal plane projection angle. Manual Therapy, 20(5), 680-685.

Herrington, L., Myer, G., \& Horsley, I. (2013). Task based rehabilitation protocol for elite athletes following Anterior Cruciate ligament reconstruction: a clinical commentary. Phys Ther Sport, 14(4), 188-198.

Hewett, T. E., Ford, K. R., Hoogenboom, B. J., \& Myer, G. D. (2010). Understanding and preventing acl injuries: current biomechanical and epidemiologic considerations - update 2010. $\mathrm{N} \mathrm{Am} \mathrm{J}$ Sports Phys Ther, 5(4), 234-251.

Hewett, T. E., Lindenfeld, T. N., Riccobene, J. V., \& Noyes, F. R. (1999). The Effect of Neuromuscular Training on the Incidence of Knee Injury in Female Athletes: A Prospective Study. The American Journal of Sports Medicine, 27(6), 699-706.

Hewett, T. E., Myer, G. D., Ford, K. R., Heidt, R. S., Colosimo, A. J., McLean, S. G., et al. (2005). Biomechanical Measures of Neuromuscular Control and Valgus Loading of the Knee Predict Anterior Cruciate Ligament Injury Risk in Female Athletes: A Prospective Study. The American Journal of Sports Medicine, 33(4), 492-501. 
Hewett, T. E., Stroupe, A. L., Nance, T. A., \& Noyes, F. R. (1996). Plyometric training in female athletes. Decreased impact forces and increased hamstring torques. Am J Sports Med, 24(6), 765-773.

Hopkins, W. G. (2002, 7th August, 2006). A scale of Magnitudes of Effect Statistics. Sportsci.org Retrieved 08/05/15, 2015, from http://sportsci.org/resource/stats/index.html

Kato, S., Urabe, Y., \& Kawamura, K. (2008). Alignment control exercise changes lower extremity movement during stop movements in female basketball players. The Knee, 15(4), 299-304.

Majewski, M., Susanne, H., \& Klaus, S. c. (2006). Epidemiology of athletic knee injuries: A 10-year study. The Knee, 13(3), 184-188.

Marinsek, M. (2010). Basic landing characteristics and their application in artistic gymnastics. Science of Gymnastics Journal, 2, 59-67.

McLean, S. G., Walker, K., Ford, K. R., Myer, G. D., Hewett, T. E., \& van den Bogert, A. J. (2005). Evaluation of a two dimensional analysis method as a screening and evaluation tool for anterior cruciate ligament injury. Br J Sports Med, 39(6), 355-362.

Munro, A., Herrington, L., \& Carolan, M. (2012). Reliability of 2-dimensional video assessment of frontal-plane dynamic knee valgus during common athletic screening tasks. J Sport Rehabil, 21(1), 7-11.

Myer, G. D., Ford, K. R., Brent, J. L., \& Hewett, T. E. (2012). An integrated approach to change the outcome part II: targeted neuromuscular training techniques to reduce identified $A C L$ injury risk factors. J Strength Cond Res, 26(8), 2272-2292.

Myer, G. D., Ford, K. R., Palumbo, J. P., \& Hewett, T. E. (2005). Neuromuscular training improves performance and lower-extremity biomechanics in female athletes. J Strength Cond Res, 19(1), 51-60.

Otsuki, R., Kuramochi, R., \& Fukubayashi, T. (2014). Effect of injury prevention training on knee mechanics in female adolescents during puberty. Int J Sports Phys Ther, 9(2), 149-156.

Paterno, M. V., Myer, G. D., Ford, K. R., \& Hewett, T. E. (2004). Neuromuscular training improves single-limb stability in young female athletes. J Orthop Sports Phys Ther, 34(6), 305-316.

Rhea, M. R. (2004). Determining the Magnitude of Treatment Effects in Strength Training Research Through the Use of the Effect Size. The Journal of Strength \& Conditioning Research, 18(4), 918-920.

Utturkar, G. M., Irribarra, L. A., Taylor, K. A., Spritzer, C. E., Taylor, D. C., Garrett, W. E., et al. (2013). The Effects of a Valgus Collapse Knee Position on In Vivo ACL Elongation. Annals of Biomedical Engineering, 41(1), 123-130.

Westermann, R. W., Giblin, M., Vaske, A., Grosso, K., \& Wolf, B. R. (2015). Evaluation of Men's and Women's Gymnastics Injuries: A 10-Year Observational Study. Sports Health, 7(2), 161-165.

Wilson, J. D., \& Davis, I. S. (2008). Utility of the Frontal Plane Projection Angle in Females With Patellofemoral Pain. Journal of Orthopaedic \& Sports Physical Therapy, 38(10), 606-615. 